\title{
DIE SAALFELDER FEENGROTTEN: EIN SCHAUBERGWERK MIT HÖHLENCHARKTER
}

\author{
Bernd Lochner*
}

\section{ZUSAMMENFASSUNG}

Die weltbekannten Saalfelder Grotten sind hervorgegangen aus einem alten Alaunschieferabbau namens "Jeremiasglück".

Der untertätige Abbau begann um 1543 und endete 1846, wobei das Alter der Tropsteine auf maximal 350 Jahre zu bemessen ist.

Die Tropfsteinbildung ist an oxidative Prozesse gebunden, was die im Vergleich zu Calcittropfsteinen sehr hohe Wachstumsgeschwindigkeit erklärt.

Seltene Minerale haben sich in den Feengrotten ausgebildet, der Hauptvertreter ist das Weichmineral Diadochit, ein Eisen (III)-phosphat wechselnder Zusammensetzung.

Die Beseitigung der Lampenflora ist sehr kompliziert, sie wird unter Zuhilfenahme grottentypischer Verbindungen realisiert.

Die Feengrotten werden derzeit von etwa 250.000 Besuchern pro Jahr aufgesucht, bisher von über 15 Millionen.

ABSTRACT [The "Saalferder Feengrotten": a show mine with cave features]

The well known"Saalferder Feengrotten" originated from an old aluminium mine called "Jeremiasglück".

The mine has been worked from 1943 to 1945; the oldest formations have an age of about 350 years. Such formations were formed by an oxidation process which explains the rather fast growth rate in comparison to the growth rate of calcite formations.

In the "Feengrotten" rare minerals can be found: the most common is a soft unstable compound, the diadochite (iron(III) phosphate).

The control of the lampenflora is rather complicate and it is obtained with some compounds normally used in caves. The "Feengrotten" are visiter yearly by about 250,000 persons and the global number of visitors up to now is more than 15 millions of persons.

Im Vorfeld des nach Norden hin steil abfallenden Thüringer Schiefergebirges liegen nahe der malerischen Stadt Saalfeld (Saale) die Weltbekannten Saalfelder Grotten.

Hervorgegangen sind diese Grotten aus einem alten Alaunschieferbergwerk namens "Jeremiasglück".

Alaunschiefer ist eine Tonschieferformation, welche sich aus organischen und anorganischen Ablagerungen am Grunde des silurischen Meeres vor etwa 410-430 Millionen Jahren gebildet hat.

Unter anaeroben Bedingungen, wie sie heute etwa noch im Schwarzen Meer vorzufinden sind, ging der organische Teil infolge der Sauerstoffarmut in Fäulnis über und setzte sich wu schwefelund kohlenstoffreichen Verbindungen um.

Durch die starken tektonischen Kräften des naheliegenden

\footnotetext{
* SaalfelderFeengrotten D-06800 SAALFELD (Saale) Deutschland
} 
Thüringer Schiefergebirge wurde die silurischen Alaunschiefer gefaltet und zerklüftet, sodaß sich mineralreiche, aufsteigende Tiefenwässer ihrer gelösten Stoffen entledigen und den Alaunschiefer nachträglich aufmineralisieren konnten.

Allgemein wird um 1540 von einem aufblühenden Alaunschieferabbau in Deutschland berichtet, nachdem man den Wert des Gesteins erkannt hatte und die Technologie der Aufbereitung beheerschte.

Um 1543 begann man im Gebiet der heutigen Feengrotten, das Gestein unter Tage abzubauen und an die Oberfläche $\mathrm{zu}$ transportieren. Dort wurde es zerkleinert, auf Lattengerüste gelegt und über zwei bis drei Jahre der Verwitterung durch Luft und Wasser ausgesetzt. Die abtropfende Flüssigkeit wurde aufgefangen, eingedampft und durch Auskristallation zu Alaun [ $\mathrm{KAl}\left(\mathrm{SO}_{4}\right)_{2} .12 \quad \mathrm{H}_{2} \mathrm{O}$ ] verarbeitet. Die verbleibende Restlauge versetzte man danach mit Eisenschrott, welcher sich auf Grund des stark sauren Charakters dieser Lösung schnell auflöste.

Durch weitere Verarbeitungsstufen erhielt man den grünen Vitriol, das Eisen (II) sulfat $\left[\mathrm{FeSO}_{4} \cdot 7 \mathrm{H}_{2} \mathrm{O}\right]$.

Um 1840 war die chemische Industrie bereits soweit entwickelt, daß sie diese begehrten Produkte schneller und billiger herstellen konnte. Somit kam der Bergbau sehr schnell zum Erliegen. Damit war das Schicksal der Grube besiegelt um 1846 fuhren die Bergleute ihre letzte Schicht.

Es wurde still im Revier, nur ein kleines Rinnsal des noch austretenden mineralreichen Grubenwassers kündete vom einst blühenden Bergbau. Doch gerade dieses Wasser war später der Anstoß zum umfangreichen wissenschaftlichen Untersuchungen, nachdem man von der einheimischen Bevölkerung Kunde erhielt über den medizinischen Wert.

So wurde um 1911 das Wasser Untersucht, und man fand die Aussagen bestätigt. Erneut stie $B$ man an gleicher Stelle in den Berg vor, um den Ursprungsort des Wassers zu erkunden.

Völlig unerwartet fand man in den alten Weitungen der ehemaligen Grube eine Faszination an Form und Farbe, die man schon auf Grund der relativ kurzen Ruhezeit von etwa 60 bis max. 350 Jahren nie für möglich gehalten hätte.

In gewissen Sinne hat sich die Natur diese Künstlich geschaffenene Hohlräume zurückerobert und mit einem höhlenartigen Charakter versehen.

Der Gedanke an eine Eröffnung als Schauobjekt lag nun sehr nahe, zumal Weihnachten 1913 der älteste und zugleich schönste Teil,der heutige Märchendom, hinzuentdeckt wurde und den späteren Touristenrundgang vorzeichnete.

Bereits ein halbes Jahr später, zu Pfingsten des Jahres 1914, wurden im Scheine umfangreicher elektrischer Beleuchtung die ersten Besucher unter Tage begrüßt. Seit dieser Zeit besuchten weit über 15 Millionen Gäste die Feengrotten. Der derzeitige Jahresdurchschnitt beläuft sich auf etwa 250.000 Besucher. 


\section{ZUR GEOLOGIE DER SAALFELDER FEENGROTTEN}

Der Saalfelder Talkessel ist in geologischer Sicht sehr vielschichtig und interessant. Dem steil aufragenden Schiefergebirge, welches sich an dieser Stelle vorzugsweise aus ordovizischen Schichten (Phycodenschiefer) zusammensetzt, ist ein silurisches Alaunschieferfeld vorgelagert mit einer Ausstrichmächtigkeit von etwa $30 \mathrm{~m}$.

Überlagert werden diese Schieferformation von obersilurischem Ockerkalk mit einigen mesovulkanischen Phophyritintrusionen. Im Alaunschiefer selbst sind Fossilien (Graptolithen) nachweisbar.

\section{ZUR CHEMIE DER FEENGROTTEN}

Den relativ leicht zu überblickenden geologischen Verhältnissen stehen in den Saalfelder Feengrotten recht komplizierte chemische Verhältnisse gegenüber, welche erst im Verlaufe langwieriger Untersuchungen ihrem Charakter nach geklärt werden konnten. Dies ist bedingt durch die im Laufe der Erdegeschichte ständig geänderten Bedingungen (Atmosphäreneinflüsse, Wasserzirkulation) und menschliche Eingriffe in den Berg (luftgefüllte Hohlräume im Wasserzirkulationsgebiet).

Ursprünglich stiegen neutrale bis alkalische Tiefenwässer ohne oxidativen Charakter bis an die Oberfläche des noch nicht mit einem Talabschnitt vershenen Vorgebirges und schützten den Alaunschiefer vor sauren und sauerstoffreichen Oberflächenwässern.

Mit der Ausbildung des naheliegenden Tales senkte sich die Mischzone langsam ab, der oberflächennahe Teil der Alaunschieferschichten war nunmehr dem langsamen Zerfall preisgegeben.

Verstärkt wurde dieser Prozeß durch den Bergbau, indem der Luftsauerstoff direkt in den Gebirgsstock eindringen und wirken konnte. Der schrittweise Zerfall und Wiederaufbau einer Reihe chemisher Verbindungen kam ab dieser Epoche ins Rollen und hält auch heute noch ungebrochen an.

Ausgangsubstanz dieser komplex verlaufenden chemischen Prozesse ist der im Alaunschiefer reichlich und sehr fein verteilt vorkommende Pyrit (Schwefeleisen), der sich unter dem Einfluß saurer Wässer leicht auflöst und zerfällt.

Hierbei bilden sich primär Eisen (II) sulfat und freie Schwefelsäure. Letztere löst in der Folge weitere Gesteinsbestandteile (insbesondere Tonerde und Phosphorit) auf.

Das Eisen (II) sulfat kann unter den gegebenen Bedingungen nur zum Teil auskristallisieren als hellgrüner Melanterit (grüner Vitriol), der überwiegende Teil setzt sich unter Einwirkung von Luft und Wasser zu Eisen (III) sulfatum.

Steigt der pH-Wert der Lösung auf über 3 bis 4 an, zerfällt auch diese Verbindung un hydrolysiert zu Eisen (III) hydroxid und Schwefelsäure.

Eine andere Umsetzungsmöglichkeit besteht darin, daB das reaktive 
Eisen (III) sulfat weiteren Pyrit auflöst und der gesamte Eisenanteil als Eisen (II) sulfat in Lösung verbleibt.

Der Schwefelanteil setzt sich um zu Schwefelsäure, der wiederum in den Prozeß eingreifen kann. Da die Schieferschichten zu einem Teil Carbonate (meist Kalk) enthalten, werden diese sofort aufgelöst und zu Gips umgesetzt. Phosphate (sie liegen im Gestein als sogenannte Phosphoritknollen vor), setzen sich ebenfalls mit Schwefelsäure um und bleiben bei genügendem Säuregrad im Wasser gelöst.

Dieses Löslichkeitsverhalten in saurer Lösung ist auch von entscheidender Beteutung für die Ausbildung von Tropfsteinen und Versinterungen.

Gelangt eine mit Eisen (II)-Verbindung angereicherte Lösung aus dem Gestein an die Oberfläche der bergbaulichen Hohlräume, so findet automatisch eine Oxidation von Eisen (II) zu Eisen (III) statt. Dabei wird ein äquivalenter Teil der Schwefelsäure chemisch gebunden, wobei der pHWert ansteigt.

Nunmehr kann ein großer Teil der in reinem Wasser oder schwach saurer Lösung unlöslichen Verbindungen ausfallen, sich als Sediment oder Trofstein ablagern (pH-Werte 4 und höher).

Das Kalk-Kohlensäure-Gleichgewicht spielt zur Tropfsteinbildung in den Feengrotten keine Rolle, da alle Carbonate bereits vorher chemisch umgesetzt wurden. All diese Sedimente und Tropfsteinmassen bestehen im wesentlichen aus dem seltenen Weichmineral "Diadochit", einem Eisensinterphosphat wechselnder Zusammensetzung.

Eine weitere Besonderheit stellt auch die Wachstumsgeschwindigkeit der Tropfsteine in den Feengrotten dar.

Unter Beachtung der bergbaulichen Abbauphasen, in der wohl keine Sedimentation von Dauer war, läßt sich das Alter' der Tropfsteine auf sechzig bis maximal 350 Jahren datieren.

Die obere und zugleich jüngste Sohle der Grotten weist Stalaktiten von etwa 15 bis $20 \mathrm{~cm}$ Länge auf, die älteste Sohle (Märchendom) solche bis $150 \mathrm{~cm}$.

Trotz schwankender Klimabedingungen und spürbaren Rückgang des Wasserangebotes (derzeit nur etwa $40 \%$ der damligen Menge!) sowie der touristischen Einflüsse hält die Sedimentbildung nach wie vor an.

Die einst zu medizinischen Zwecken genutzten Quellen sind derzeit versiegt. Ihre Zusammensetzung entsprach etwa der, wie sie heute noch in den Heilquellen von Levico-Vetriolo bei Trient zu verzeichnen ist.

Die Saalfelder Heilquellen, entsprungen in den Feengrotten, waren damals Deutschlands einzige Eisen-Arsen-Sulfat-PhosphatQuellen und auf Grund ihrer seltenen Zusammensetzung eine sehr begehrte Medizin.

\section{LAMPENFLORA IN DEN FEENGROTTEN}

Bedingt durch den nicht abreißenden Besucherstrom sowie durch technische Einflüsse (Beleuchtung, Belüftung) hat sich in den letzten Jahren in zunehmendem Maße eine sehr resistente Lampenflora 
angesiedelt. Einige Spezies sind außer in den Feengrotten in keiner anderen deutschen Schauhöhle oder einem Schaubergwerk zu finden!

Die besondere Gefahr dieser Vergrünung bestand darin, daß über Jahre kein großer Einfluß auf die Verminderung des Wachstums ausgeübt wurde und darüber hinaus mit der Lampenflorabeseitigung in Kalkhöhlen nicht verglichen werden konnte.

Einzelne Tropfsteine hatten bereits den dreifachen Durchmesser durch Moosablagerungen angenommen, eine mechanische Beseitigung war nich möglich.

Somit verblieb als einziger Weg nur noch eine vorsichitige chemische Behandlung unter Beachtung folgender Grundsätze:

- die befallenen Flächen sind sehr empfindlich, können also mechanisch nicht behandelt werden

- die Anwendung handelsüblicher Biocide ist aus bestimmten Umweltgründen nicht gestattet.

- die Behandlung muB schadlos wiederholbar sein und darf die Tropfsteine in Form und Farbe nicht verändern.

- die eingesetzen Mittel müssen lange Wirksamkeit besitzen und dürfen nich abdriften.

Somit gelangten nur solche Verbindungen zum Einsatz, die auch in der Natur der Feengrotten nachgewiesen und bei entsprechender Konzentration wirksam genug waren.

Es handelt sich um folgende Substanzen:

- $\mathrm{KAl}\left(\mathrm{SO}_{4}\right)_{2} \cdot 12 \mathrm{H}_{2} \mathrm{O} \quad$ (Alaun, Kalialaun)

- $\mathrm{FeSO}_{4} .7 \mathrm{H}_{2} \mathrm{O}$ (Eisenvitriol, Eisen (II) sulfat)

- $\mathrm{CuSO}_{4} \cdot 5 \mathrm{H}_{2} \mathrm{O} \quad$ (Kupfervitriol, Kupfersulfat)

Durch Vernebeln der 0,5 bis $5 \%$ starken Lösungen gelang es im Verlaufe mehrerer Monate, die Lampenflora schrittweise zu beseitigen und den ursprünglichen Zustand größtenteils wieder herzustelen.

Die grünliche Färbung der bereits abgestorbenen Pflanzenteile konnte mit einer verdünnte $\mathrm{H}_{2} \mathrm{O}_{2}$ - Lösung (max. 5\%) beseitigt werden.

Nach einer Anwendungszeit von nunmehr etwa drei Jahren kann eingeschätzt werden, $\mathrm{da} B$ der eingeschlagene Weg richtig ist und den Feengrotten nicht geschadet hat.

Somit gelingt es, die Saalfelder Feengrotten dem Besucher wieder so zu zeigen, wie es mit der Eröffnung im Jahre 1914 möglich war. 